\title{
One-dimensional electronic properties of the Bechgaard salts
}

\author{
K. PENC and F. MILA \\ Institut de Physique, Université de Neuchâtel, 1 rue Breguet, 2000 Neuchâtel, Switzerland
}

\begin{abstract}
An estimate of the gap $\Delta_{\rho}$ that opens at the Fermi level in a 3/4-filled Hubbard model with alternating hopping integrals is obtained on the basis of weak and strong coupling results. Assuming that this gap will induce a minimum of resistivity at a temperature $T_{\rho} \simeq \Delta_{\rho} / \pi$, we show that the hopping integrals provided by quantum chemistry calculations based on structural data are in reasonable agreement with the experimental resistivity curves if $U$ is the same for all compounds $(\simeq 850 \mathrm{meV})$. In particular, very small values can be obtained for the TMTSF series without assuming that the dimerization is itself very small. The discrepancies between this model and the magnetic data are briefly discussed.
\end{abstract}

The electronic structure of the Bechgaard salts is still an active and controversial subject [1]. Even above the cross-over temperature $T_{x}$ (of order $30 \mathrm{~K}$ ) where the properties are believed to be essentially one-dimensional there is no general agreement on the effective model that one should use to describe them. For instance, it is not clear whether (TMTSF) ${ }_{2} \mathrm{PF}_{6}$ should be considered as a $1 / 2$-filled or as a $3 / 4$-filled system in this temperature range, and this is a crucial issue in understanding photoemission data recently obtained on that compound [2]. In particular, the ground-state can be a Luttinger liquid is the system if $3 / 4$ filled [3], but not if it is $1 / 2$ filled. More precisely, it is generally believed that a 3/4-filled Hubbard model with alternating hopping amplitudes should be a reasonable starting point, but the values of the parameters that one should consider for different members of the series is still largely unknown. Such a model is described by the Hamiltonian

$$
\begin{array}{r}
H=-t_{1} \sum_{i \text { even, } \sigma}\left(c_{i, \sigma}^{\dagger} c_{i+1, \sigma}+\text { h.c. }\right) \\
-t_{2} \sum_{\text {iodd }, \sigma}\left(c_{i, \sigma}^{\dagger} c_{i+1, \sigma}+\text { h.c. }\right)+U \sum_{i} n_{i \uparrow} n_{i \downarrow}
\end{array}
$$

where $U$ is the on-site Coulomb repulsion and $n_{i, \sigma}=c_{i, \sigma}^{\dagger} c_{i, \sigma}$. The operators $c_{i, \sigma}^{\dagger}$ create particles in the HOMO of TMTSF or TMTTF with $\operatorname{spin} \sigma$. We have included two hopping integrals $t_{1}$ and $t_{2}\left(t_{1}>t_{2}\right)$ to describe the dimerization along the stacks. From chemistry considerations one knows that there are 3 electrons in these HOMO for each pair (TMTSF) $)_{2}$ or (TMTTF $)_{2}$, so that the system is $3 / 4$ filled. However, the alternating hoppings will open a dimerization gap $\Delta_{D}=2\left(t_{1}-t_{2}\right)$. In this picture the model become effectively half-filled when $T<\Delta_{D}$ and the ground-state has a SDW instability.

We have essentially three sources of information on these parameters. The first one comes from X-ray and neutron measurements which have provided accurate values for the structural parameters of these compounds [4]. Using them as inputs, quantum chemistry calculations [5] have provided values for various hopping integrals, in particular for the hopping integrals along the stacks $t_{1}$ and $t_{2}$, for a few compounds (see table 1). The integrals relative to TMTSF compounds are roughly twice as large as those relative to TMTTF compounds mainly because the selenium $4 p$ orbitals are more extended than the sulfur $3 p$ ones. Although the actual value of the repulsion $U$ is difficult to get from quantum chemistry calculations, various theoretical estimates of this parameter suggest that that it depends very little on the compound, and that it is rather smaller for TMTSF than for TMTTF, again due to the spatial extension of the orbitals.

The second source of information comes from the extensive magnetic measurements performed 


\begin{tabular}{|lcccc|}
\hline Compound & $t_{1}(\mathrm{meV})$ & $t_{2}(\mathrm{meV})$ & $\Delta_{D} / D$ & $T_{\rho}(\mathrm{K})$ \\
\hline \hline (TMTTF $)_{2} \mathrm{PF}_{6}$ & 137 & $93(300 \mathrm{~K})$ & 0.191 & $\simeq 220$ \\
\hline (TMTTF $)_{2} \mathrm{Br}$ & 133 & $119(300 \mathrm{~K})$ & 0.056 & $\simeq 100$ \\
\hline (TMTSF $)_{2} \mathrm{PF}_{6}$ & 280 & $254(4 \mathrm{~K})$ & 0.049 & $<T_{x} \simeq 30 \mathrm{~K}$ \\
\hline (TMTSF $)_{2} \mathrm{ClO}_{4}$ & 287 & $266(7 \mathrm{~K})$ & 0.038 & $<T_{x} \simeq 30 \mathrm{~K}$ \\
\hline
\end{tabular}

Table 1: Some data for a few Bechgaard salts

mainly by the Orsay group [6]. The temperature and pressure dependence of the susceptibility can be understood within a model of interacting electrons if $E_{F} \simeq 3100 \mathrm{~K}$ for TMTSF and $1600 \mathrm{~K}$ for TMTTF compounds, while $g_{1}$ (backscattering energy) should be of order $\pi v_{F}$ in each case. For the model of eq. (1), $g_{1}$ is of order $U / 2$, so that $U$ should be twice as large for TMTSF than for TMTTF.

Finally, some information can in principle be deduced from the resistivity measurements. The members of the TMTTF series exhibit a resistivity minimum at relatively high temperatures $\left(T_{\rho} \simeq 100-220 K\right)$, which presumably comes from a gap $\Delta_{\rho} \simeq \pi T_{\rho}$ at the Fermi level. On the other hand, no resistivity minimum has been detected in the metallic phase of TMTSF compounds which means that the gap (if any) must be very small.

If one tries to put all this information together, one is faced with at least two difficulties. First, magnetic measurements lead to a $U$ twice as large for TMTSF than for TMTTF, while simple considerations based on the extension of the orbitals (and theoretical estimates) suggest that the repulsion can only be smaller for TMTSF than for TMTTF. Second, if $U / \pi v_{F}$ is the same in both materials, the only way to explain why the gap is one to two orders of magnitude smaller in TMTSF than in TMTTF is to assume that $t_{1}$ and $t_{2}$ are almost equal in TMTSF, in contradiction with quantum chemistry calculations.

How can we reconcile these facts? The first step is to check whether it is possible to get gaps at the Fermi level that differ by orders of magnitude with the assumption that $U$ varies very little from one compound to the other and that the hopping integrals have the values predicted by quantum chemistry calculations. To perform this check, we need a quantitative estimate of the gap that opens at the Fermi level as a function of $t_{1}, t_{2}$ and $U$.

When $t_{1}$ and $t_{2}$ are different, the model of eq. (1) cannot be solved by Bethe ansatz, and it turns out to be a very difficult task to get a reliable, quantitative estimate of $\Delta_{\rho}$ for all $U$. All what we have been able to do so far is to get good estimates of $\Delta_{\rho}$ in various limits. When $U$ is small, we can use the renormalization group equations of $g$-ology $[7,8]$. In the case of a halffilled band, the physics is governed by

$$
\begin{gathered}
\frac{\mathrm{d}\left(\tilde{g}_{1}-2 \tilde{g}_{2}\right)}{\mathrm{d} \ln S}=\tilde{g}_{3}^{2}+\frac{1}{2}\left(\tilde{g}_{1}-2 \tilde{g}_{2}\right) \tilde{g}_{3}^{2} \\
\frac{\mathrm{d} \tilde{g}_{3}}{\mathrm{~d} \ln S}=\left(\tilde{g}_{1}-2 \tilde{g}_{2}\right) \tilde{g}_{3}+\frac{1}{4}\left[\left(\tilde{g}_{1}-2 \tilde{g}_{2}\right)^{2}+\tilde{g}_{3}^{2}\right] \tilde{g}_{3}
\end{gathered}
$$

where $S$ is a momentum cut-off, $\tilde{g}_{i}=g_{i} / \pi v_{\rho}$, $v_{\rho}=v_{F}+g_{4} / 2 \pi$ is the velocity of charge excitation and $g_{1}, g_{2}, g_{3}$ and $g_{4}$ are the amplitudes of the various scattering processes [7]. In our model, after diagonalizing the hopping term, the scattering amplitudes near the Fermi level are given by

$$
\begin{gathered}
g_{1}=g_{2}=g_{4}=\frac{U}{2} \\
g_{3}=\frac{U}{2} \frac{\Delta_{D}}{D} \frac{2}{1+\left(\Delta_{D} / D\right)^{2}}
\end{gathered}
$$

and the Fermi velocity is given by

$$
v_{F}=\frac{1}{8} \frac{D^{2}-\Delta_{D}^{2}}{\sqrt{\left(D^{2}+\Delta_{D}^{2}\right) / 2}}
$$

where $D=2\left(t_{1}+t_{2}\right)$ is the total bandwidth and $\Delta_{D}=2\left(t_{1}-t_{2}\right)$ is the dimerization gap. In this model we can explicitly see the dependence of the umklapp scattering $g_{3}$ on the dimerization gap, as proposed in [9].

To obtain a quantitative estimate of $\Delta_{\rho}$, one can proceed in two steps. First, one can get the 
functional dependence of $\Delta_{\rho}$ on the $g$ 's by integrating eqs. (2) and (3). This yields

$$
\ln \frac{\Delta_{\rho}}{v_{F}} \simeq\left(\frac{1}{4}-\frac{1}{\tilde{g}}\right) \frac{\operatorname{Arth} \sqrt{1-\xi^{2}}}{\sqrt{1-\xi^{2}}}+\frac{1}{2} \ln \xi \tilde{g}+C
$$

where $\tilde{g}=2 \tilde{g}_{2}-\tilde{g}_{1}, \xi=\tilde{g}_{3} / \tilde{g}$ and $C$ is a constant to be determined. This expression is valid for all $\xi$ and generalizes the well known formulae that can be found in the literature in the cases $\xi \ll 1$ and $\xi=1[8]$.

Then, one can determine the constant $C$ by looking at a special case for which the exact form (including the prefactor) is available [10]. When $t_{2}, U \ll t_{1}$, the bonding and antibonding states on neighbouring sites with hopping $t_{1}$ form two bands separated by a large energy of order $2 t_{1}$. The Hamiltonian becomes equivalent to a Hubbard model with on-site repulsion $U / 2$ and effective hopping $t_{2} / 2$. In this case we know the exact result for the charge gap from the Lieb-Wu equations [11] from which we get

$$
\Delta_{\rho}= \begin{cases}\left(8 t_{2} / 2 \pi\right) \sqrt{U / t_{2}} \mathrm{e}^{-2 \pi t_{2} / U} & \text { if } U \ll t_{2} \\ U / 2-2 t_{2} & \text { if } U \gg t_{2}\end{cases}
$$

The first expression $\left(U \ll t_{2}\right)$ should be equivalent to the expression of eq. (7) in the limit $\xi=1$ which yields $C=1 / 4+\ln (4 \sqrt{2 / \pi})$.

So, our final expression for the gap in the small $U$ limit reads

$$
\begin{aligned}
\Delta_{\rho}= & \frac{4 v_{F}}{\pi} \sqrt{\frac{\xi U}{v_{F}}} \exp \left(-\frac{1}{4} \frac{\operatorname{Arth} \sqrt{1-\xi^{2}}}{\sqrt{1-\xi^{2}}}+\frac{1}{4}\right) \\
& \times \exp \left(-\frac{2 \pi}{U} \frac{\operatorname{Arth} \sqrt{1-\xi^{2}}}{\sqrt{1-\xi^{2}}}\right)
\end{aligned}
$$

For the model of eq. (1), $\xi$ depends only on $\Delta_{D} / D$ through $\xi=2\left(\Delta_{D} / D\right) /\left(1+\left(\Delta_{D} / D\right)^{2}\right)$. Plots of $\Delta_{\rho}$ as a function of $U$ are given in fig. 1 for the values of $\Delta_{D} / D$ listed in table 1 . This approximation should remain accurate to within $10 \%$ as long as $U<4 v_{F} \operatorname{Arth} \sqrt{1-\xi^{2}} / \sqrt{1-\xi^{2}}$ (solid curves).

When $U$ is infinite, it is more convenient to think in terms of holes. The charge degrees of freedom can then be described by a tight-binding Hamiltonian of spinless fermions with alternating hopping amplitudes $-t_{1}$ and $-t_{2}$, and the gap is given by $\Delta_{\rho}=\Delta_{D}=2\left(t_{1}-t_{2}\right)$. As can

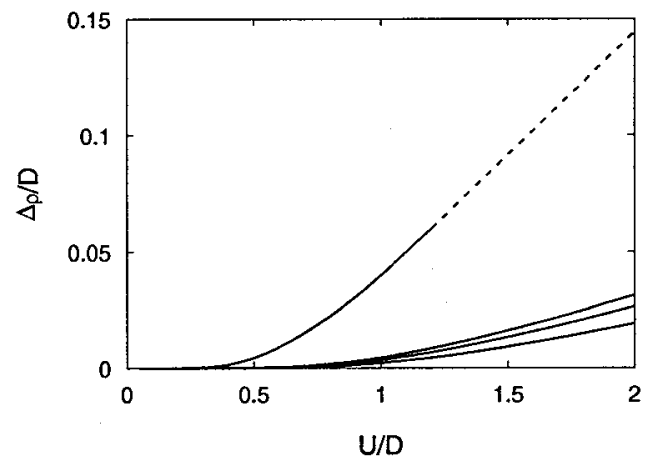

Figure 1: The charge gap $\Delta_{\rho}$ as a function of $U$ for different values of $\Delta_{D} / D(0.191,0.056,0.049$ and 0.038 from top to bottom).

be seen from fig. (1), the largest reliable value obtained in the weak coupling limit is not too far from the limiting value obtained for $U$ infinite, and we expect the exact curve not to be too far from the curve obtained by extrapolating our weak-coupling results up to this limiting value. More work is needed however to justify this assumption.

Coming back to our original problem, we now have a reasonable approximation of the gap $\Delta_{\rho}$ as a function of $U$ for the compounds listed in table 1 assuming that the hopping integrals are those given by quantum chemistry calculations. Two effects combine to give smaller values for TMTSF than TMTTF: the dimerization, although not negligible, is smaller for TMTSF, and more importantly the effective repulsion $U / D$ is smaller because $D$ is larger for TMTSF while the bare repulsion $U$ is about the same in both cases. It turns our that these effects, when combined, can yield huge differences in the values of $\Delta_{\rho}$. To see this, let us start by estimating $U$ for (TMTTF $)_{2} \mathrm{PF}_{6}$. In that case, $\Delta_{D} / D \simeq 0.19$ (upper curve) and $\Delta_{\rho} / D \simeq 0.13$, so that $U / D$ should be equal to 1.86 , i.e. $U \simeq 854 \mathrm{meV}$. Using this value of $U$ for the other compounds yields $\Delta_{\rho} \simeq 16 \mathrm{~K}$ for $(\mathrm{TMTSF})_{2} \mathrm{PF}_{6}, \Delta_{\rho} \simeq 8.4 \mathrm{~K}$ for $(\mathrm{TMTSF})_{2} \mathrm{ClO}_{4}$, and $\Delta_{\rho} \simeq 125 \mathrm{~K}$ for $(\mathrm{TMTTF})_{2} \mathrm{Br}$. These results are in reasonable agreement with the resistivity measurements (remember that $\Delta_{\rho} \simeq \pi T_{\rho}$ ). In 
particular, there is no problem in getting gaps that are very small for the TMTSF series. The disagreement for (TMTTF) ${ }_{2} \mathrm{Br}$ might be due to the fact that we have values of the hopping integrals at $300 \mathrm{~K}$, while the resistivity minimum occurs at $100 \mathrm{~K}$. In fact, to be able to make a truly quantitative analysis, we should have data at $220 \mathrm{~K}$ for (TMTTF) ${ }_{2} \mathrm{PF}_{6}$. In any case, in spite of these uncertainties, we believe that the main point is clearly established, namely that very different gaps can be obtained in each series without assuming that the dimerization is very small in the TMTSF one.

In conclusion, our analysis of the resistivity minima in terms of the model of eq. (1) leads to encouraging results about the validity of the theoretical estimates of $t_{1}, t_{2}$ and $U$. This analysis should be considered as preliminary, and more work is needed to get reliable estimates of $\Delta_{\rho}$ for intermediate values of $U$. However, if the results of the present work are to be confirmed, we believe that the model of eq. (1) with the parameters of table 1 and $U \simeq 850 \mathrm{meV}$ is a reasonable candidate to describe the electronic properties of the Bechgaard salts, and one should think about accurate ways of calculating the magnetic properties of that model to see if they can be reconciled with the magnetic measurements.

The authors are grateful to D. Baeriswyl, M. Dzierzawa, E. Jekelmann, D. Jérôme, J. P. Pouget, H. J. Schulz, P. Wzyetek and X. Zotos for valuable discussions on this topic.

\section{References}

[1] For an early review, see D. Jérôme and $H$. Schulz, Adv. Phys. 31 (1982) 299; for a more recent review, see Y. Firsov, V. Prigodin and C. Seidel, Phys. Rep. 126 (1985) 245.

[2] B. Dardel, D. Malterre, M. Grioni, P. Weibel, Y. Baer, J. Voit, D. Jérôme, preprint.

[3] F. Mila and X. Zotos, preprint.

[4] See B. Gallois et al, Acta Cryst. B42 (1986) 564 , and reference therein.

[5] L. Ducasse et al, J. Phys. C 19 (1986) 3805.
[6] For a recent review, see P. Wzietek et al, $J$. Phys. I France 3 (1993) 171, and references therein.

[7] J. Sólyom , Adv. Phys. 28 (1979) 201.

[8] V. J. Emery, Highly Conducting OneDimensional Solids, J. T. Devreese, R. P. Edvard and V. E. van Doren Eds. (Plenum, New York, N. Y., 1979) p. 1221.

[9] S. Barisic and S. Brazovskii, Recent Developments in Condensed Matter Physics, Vol. 1, J. T. Devreese Ed. (Plenum, New York, N. Y., 1981) p. 327.

[10] A. I. Larkin and J. Sak, Phys. Rev. Lett. 39 (1977) 1025.

[11] E. H. Lieb E. H. and F. Y. Wu, Phys. Rev. Lett. 20 (1968) 1445. 